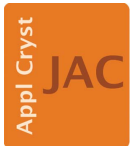

JOURNAL OF

APPLIED

CRYSTALLOGRAPHY

ISSN 1600-5767

Received 11 February 2015

Accepted 21 May 2015

Edited by G. Kostorz, ETH Zurich, Switzerland

Keywords: quasi-mosaicity; hard X-ray focalization; charged-particle beam steering.

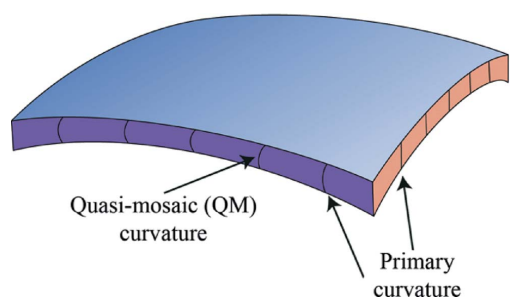

C 2015 International Union of Crystallography

\section{The 'quasi-mosaic' effect in crystals and its applications in modern physics}

\author{
Riccardo Camattari, Vincenzo Guidi,* Valerio Bellucci and Andrea Mazzolari
}

Department of Physics and Earth Sciences, University of Ferrara, Via Saragat 1/C, 44122 Ferrara, and INFN, Section of Ferrara, Italy. *Correspondence e-mail: guidi@fe.infn.it

'Quasi-mosaicity' is an effect of anisotropy in crystals that permits one to obtain a curvature of internal crystallographic planes that would be flat otherwise. The term 'quasi-mosaicity' was introduced by O. Sumbaev in 1957. The concept of 'quasi-mosaicity' was then retrieved about ten years ago and was applied to steering of charged-particle beams at the Super Proton Synchrotron at CERN. Beams were deviated by exploiting channeling and volume reflection phenomena in curved crystals that show the 'quasi-mosaic' effect. More recently, a crystal of this kind was installed in the Large Hadron Collider at CERN for beam collimation by the UA9 collaboration. Since 2011, another important application involving the 'quasi-mosaic' effect has been the focalization of hard X-rays and soft $\gamma$-rays. In particular, the possibility of obtaining both high diffraction efficiency and the focalization of a diffracted beam has been proved, which cannot be obtained using traditional diffracting crystals. A comprehensive survey of the physical properties of 'quasi-mosaicity' is reported here. Finally, experimental demonstrations for adjustable values of the 'quasi-mosaic' curvature are provided.

\section{Introduction}

Bent crystals can be advantageously employed for several applications. Curved crystals are used in synchrotrons as highefficiency monochromators for high-energy X-ray beamlines (Schulze et al., 1998; Suortti et al., 1997) and to control neutron diffraction with wide angular acceptance (Rekveldt, 1983; Rekveldt \& Westerhuuijs, 1987; Mikula et al., 1990; Popovici et al., 1999). Owing to the strong electric field generated by the ordered atoms in a crystal, it is possible to manipulate charged-particle trajectories via coherent effects such as channeling and volume reflection (Tsyganov, 1976b; Taratin \& Vorobiev, 1987). Bent crystals have already been proposed to be used in collimation systems (Maslov et al., 1991) and demonstrated to work as a primary collimator for beams at the Tevatron (Shiltsev et al., 2010), SPS (Scandale et al., 2010) and U-70 (Afonin et al., 2011) accelerators. In addition, bent crystals have been adopted for beam steering (Elishev et al., 1979) and extraction (Bellazzini et al., 1991; Akbari et al., 1993; Afonin et al., 2001; Carrigan et al., 2002; Fliller et al., 2005) in circular accelerators, as well as for splitting and focusing of external beams (Denisov et al., 1992). Moreover, crystalassisted collimation and extraction of TeV-energy ion beams have been proposed as upgrades of the Large Hadron Collider (LHC) (Scandale et al., 2011; Brodsky et al., 2013). Radiation emission due to curved trajectories of charged particles in bent crystals was studied in order to yield photon production through bremsstrahlung, channeling radiation, parametric $\mathrm{X}$-ray radiation and undulator use (Korol et al., 2004). Bent crystals were tested as optical elements for focusing hard X- 
and $\gamma$-rays with high efficiency and resolution (Barrière et al., 2010; Guidi, Barrière et al., 2011). Such bent crystals may represent the solution that the astrophysics community has sought for realizing experiments based on X-ray concentrators (Von Ballmoos, 2013). An X-ray concentrator can also be used for high-quality imaging in nuclear medicine (Roa et al., 2005; Paternò et al., 2015). In particular, such an imager would improve $\gamma$-ray detection in single-photon emission computed tomography and in positron emission tomography by providing better scan resolution. This, in turn, would lead to a lower radioactive dose being administered to the patient (Smither et al., 2005).

The possibility of controlling the complete deformation field within a crystal has to be realized for further improvement of the aforementioned and other applications. When a specific moment is applied to a crystalline material, some secondary curvatures may arise within the solid. A well known secondary deformation is the anticlastic curvature that occurs in a medium subjected to two moments as a consequence of the differential lateral contraction caused by the Poisson effect. In particular, it occurs in the perpendicular direction with respect to the principal curvature. When the two curvatures are combined, the deformed crystal takes the shape of a saddle (see Fig. 1a).

Owing to their periodic structure, the physical properties of crystals may be anisotropic. A secondary deformation caused by anisotropic effects is the 'quasi-mosaic' (QM) curvature, which was first explained quantitatively by O. Sumbaev in 1957 in terms of crystal anisotropy (Sumbaev, 1957). QM crystals ${ }^{1}$ belong to a class of bent crystals featuring two curvatures of two orthogonal crystallographic planes (see Fig. 1b). As a crystal is bent to a primary curvature by external forces, a secondary curvature can be generated within the crystal, i.e. the QM curvature (Ivanov et al., 2005). This curvature is always absent in an isotropic material. The planes bent by the QM effect are orthogonal to the main surface of the plate. 'Quasi-mosaicity' is a mechanical property driven by anisotropy and is fully explained by the theory of linear elasticity in an anisotropic medium (Lekhnitskii et al., 1956).

The choice of the name 'quasi-mosaicity' dates back to its discovery. Though the term 'quasi-mosaic crystal' may sound like the term 'mosaic crystal' (Zachariasen, 1945), the two types of crystals are significantly different. A mosaic crystal is a polycrystalline material, consisting of an ensemble of small perfect crystals - the crystallites - with their crystallographic orientations spread around a nominal direction like the small tiles in ancient artistic mosaics. The crystallites of a mosaic crystal are slightly misaligned with each other, with a Gaussian function as angular distribution. As a consequence, the Bragg condition can be met for different photon energies. Thus, mosaic crystals have been used to enlarge the energy passband and increase the efficiency in diffraction experiments. In contrast, a QM crystal is a monocrystalline material characterized by bent crystallographic planes along particular

\footnotetext{
${ }^{\mathbf{1}}$ Hereinafter, by 'quasi-mosaic' crystals, we mean crystals that present crystallographic planes bent because of the 'quasi-mosaic' effect.
}

directions. However, as is clarified here below, its response under X-ray diffraction may resemble that of a mosaic crystal.

Indeed, in 1950, a study on X-ray diffraction in singlecrystal quartz plates elastically bent to a cylindrical form revealed an anomalous increase in the integrated diffraction efficiency and in the energy passband (Lind et al., 1950). This phenomenon was interpreted as an increase in the misalignment of the crystallites. However, this effect vanished as the bending was removed. Actually, the increase in integrated diffraction efficiency and the enlargement of the energy passband were due to the bending of the diffracting planes owing to the QM effect. Because of this first misunderstanding, the term 'quasi-mosaicity' was coined, because the crystal, once bent, behaved as a mosaic crystal (Sumbaev, 1968).

QM crystals have been employed for steering chargedparticle beams. A crystal of this kind was installed in the LHC at CERN for beam collimation by the UA9 collaboration (Scandale et al., 2011). Moreover, QM crystals are particularly suitable for the manipulation of negatively charged particles and low-energy positively charged particles, where thin crystals are needed. Indeed, as explained in $\S 5$, QM crystals can be very thin in the direction of the particle beam, because the curved planes used to steer the charged particles are orthogonal to the major surface of the crystal.

QM crystals have also been proposed to be used for the focalization of hard X-rays as part of ASI's Laue project (Camattari, Battelli et al., 2013; Camattari, Paternò, Battelli et al., 2014); this project consists of the implementation of a Laue lens prototype for hard X-ray and soft $\gamma$-ray astronomy (Virgilli et al., 2013; Liccardo et al., 2014). A Laue lens with QM crystals is an arrangement of curved plates whose primary curvature lies on a spherical calotte of radius $R$, while the QM curvature allows high-efficiency diffraction from curved diffracting planes (Authier, 2001). Owing to Bragg diffraction, focusing of each QM sample converges on a focal spot at a distance $f=R / 2$ on the symmetry axis of the calotte. Most importantly, 'quasi-mosaicity' allows focusing of a photon flux

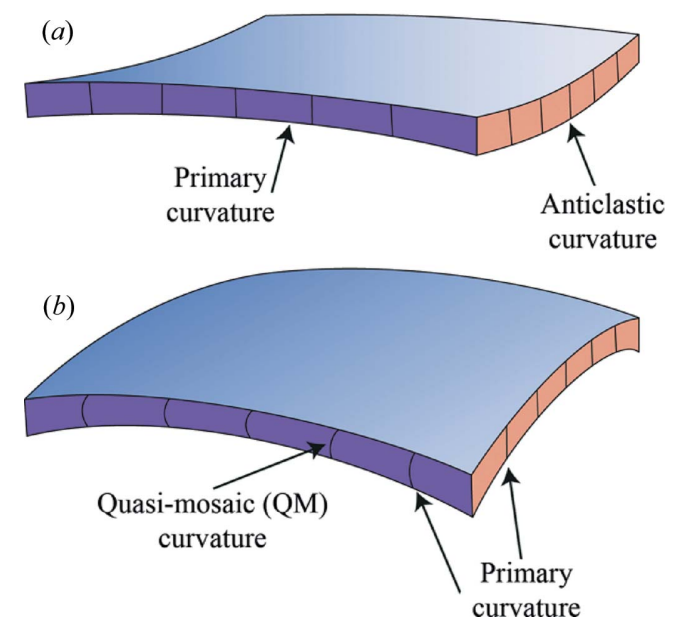

Figure 1

(a) Schematic representation of a plate with anticlastic deformation. (b) Deformed plate with 'quasi-mosaic' curvature. 
in a spot smaller than the size of the diffracting crystal through bent diffracting planes, i.e. with high integrated diffraction efficiency.

This is in contrast to diffraction by a traditional mosaic or bent crystal, the spot of which is no smaller than the crystal size exposed to the photons. For a QM crystal, the primary curvature is responsible for focusing the photon flux, while the QM curvature increases the integrated diffraction efficiency (Guidi, Bellucci et al., 2011). As a result, the sensitivity of a Laue lens could be increased (Bellucci et al., 2013; Camattari, Paternò, Bellucci \& Guidi, 2014). The concept of QM crystals in a Laue lens is explained in $\S 6$.

In this paper, we provide a comprehensive review of the QM effect in crystals. The calculations of the QM curvature for the cases of $\mathrm{Si}$ and $\mathrm{Ge}$ crystals are shown in $\S 3$. In $\S 4$, some
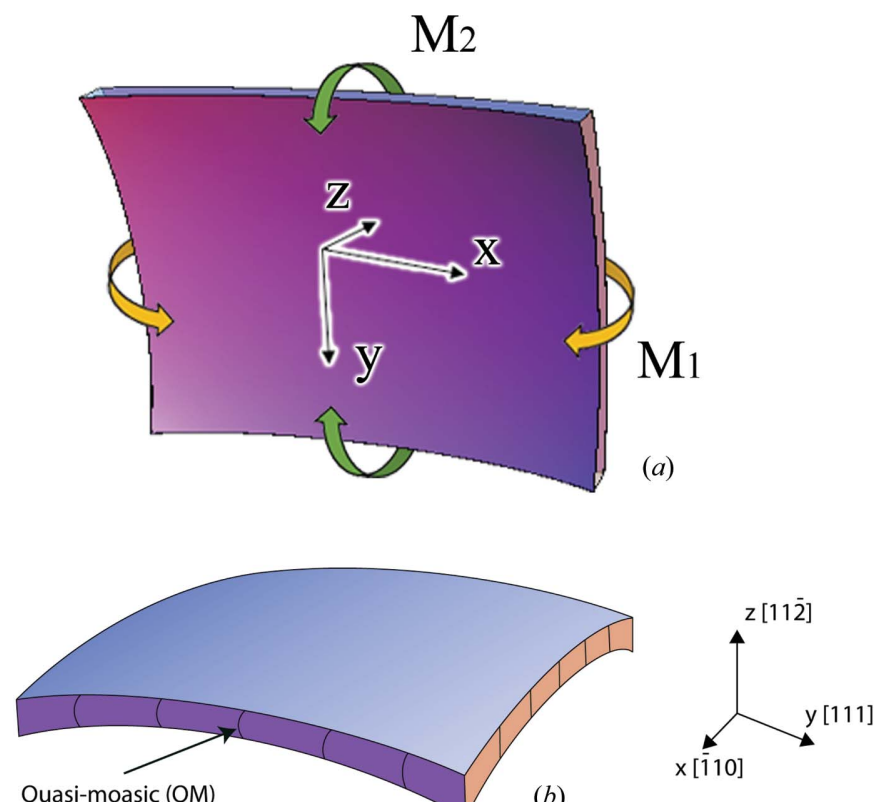

Quasi-moasic (QM)

(b)
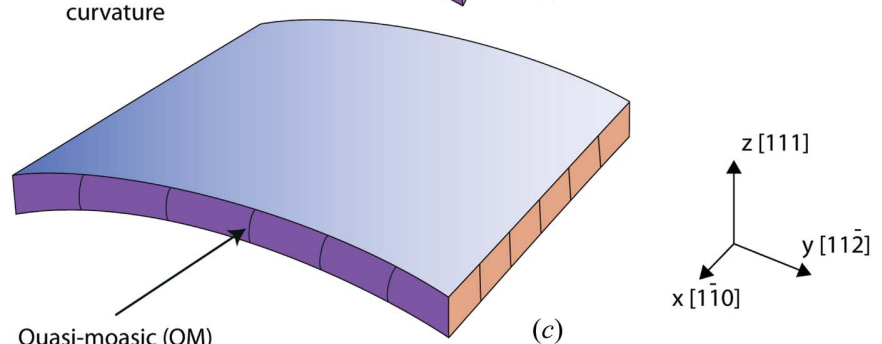

Quasi-moasic (QM)

(c)
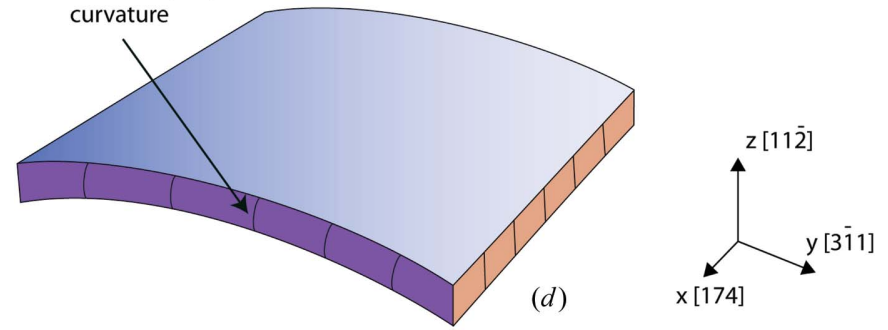

Figure 2

Schematic representation of a crystal plate with the coordinate system used for the modeling. In $(a)$, the bent arrows symbolize applied moments $M_{1}$ and $M_{2}$. In $(b)$, two moments are applied, while in $(c)$ and $(d)$, a single moment is applied. The crystallographic orientation and QM curvature for the three cases are highlighted. techniques to fabricate QM crystals are reported. In $\S \S 5$ and 6, a review of the applications involving QM crystals is presented. In addition, practical examples of the steering of charged-particle beams and focusing of hard X-rays are reported. Finally, experimental demonstrations of large and adjustable QM curvature are given in $§ 7$.

\section{Bent crystalline plates in the theory of linear elasticity}

As a consequence of external forces, a crystal can be deformed. The curvature of the crystallographic planes in a bent crystal can be calculated through the displacement field as a function of $u(\mathbf{r}), v(\mathbf{r})$ and $w(\mathbf{r})$, which are deformations along the $x, y$ and $z$ axes, respectively. The normal $(\sigma)$ and tangential $(\tau)$ components of the stress tensor are bound up with the mechanical moments $M_{1}$ and $M_{2}$ applied to the crystal as

$$
\begin{gathered}
\sigma_{x}=\frac{M_{1}}{I_{x}} z, \quad \sigma_{y}=\frac{M_{2}}{I_{y}} z, \quad \sigma_{z}=0, \\
\tau_{y z}=0, \quad \tau_{x z}=0, \quad \tau_{x y}=0,
\end{gathered}
$$

where $I_{x}$ and $I_{y}$ are the moments of inertia. Fig. 2(a) shows a plate on which two couples of moments are applied.

From the theory of homogeneous anisotropic thin plates subjected to bending with the following boundary conditions,

$$
\begin{aligned}
\left.\frac{\mathrm{d} w}{\mathrm{~d} x}\right|_{0}=\left.\frac{\mathrm{d} w}{\mathrm{~d} y}\right|_{0} & =0, \quad \frac{\mathrm{d} v}{\mathrm{~d} x}-\frac{\mathrm{d} u}{\mathrm{~d} y}=0, \\
u(0) & =v(0)=w(0)=0
\end{aligned}
$$

it is known that the displacement field arising from the crystal plate deformation is

$$
\begin{aligned}
u= & \frac{1}{2}\left[\frac{M_{1}}{I_{x}}\left(S_{51} z^{2}+S_{61} y z+2 S_{11} x z\right)\right. \\
& \left.+\frac{M_{2}}{I_{y}}\left(S_{52} z^{2}+S_{62} y z+2 S_{12} x z\right)\right], \\
v= & \frac{1}{2}\left[\frac{M_{1}}{I_{x}}\left(S_{41} z^{2}+2 S_{21} y z+S_{61} x z\right)\right. \\
& \left.+\frac{M_{2}}{I_{y}}\left(S_{42} z^{2}+2 S_{22} y z+S_{62} x z\right)\right], \\
w= & \frac{1}{2}\left[\frac { M _ { 1 } } { I _ { x } } \left(S_{31} z^{2}-S_{11} x^{2}-S_{12} y^{2}-S_{16} x y\right.\right. \\
& \left.+\frac{M_{2}}{I_{y}}\left(S_{32} z^{2}-S_{12} x^{2}-S_{22} y^{2}-S_{26} x y\right)\right],
\end{aligned}
$$

where $S_{i j}$ are the components of the compliance tensor for an anisotropic material referred to the $(x, y, z)$ Cartesian system (Lekhnitskii et al., 1956; Lekhnitskii, 1981). The relationship between the curvature of the middle plane of the plate and bending moments $M_{1}$ and $M_{2}$ (per unit length), uniformly distributed along its sides, is 


$$
\begin{aligned}
& \frac{1}{R_{x}}=\frac{\partial^{2} w}{\partial x^{2}}=-\frac{6}{h_{\mathrm{s}}^{3}}\left(2 M_{1} S_{11}+2 M_{2} S_{12}\right), \\
& \frac{1}{R_{y}}=\frac{\partial^{2} w}{\partial y^{2}}=-\frac{6}{h_{\mathrm{s}}^{3}}\left(2 M_{1} S_{12}+2 M_{2} S_{22}\right),
\end{aligned}
$$

where $h_{\mathrm{s}}$ is the plate thickness, and $R_{x}$ and $R_{y}$ are the radius of the primary curvature along the $x$ and $y$ axis, respectively.

Starting from equation (3), if we apply two moments $M_{1}$ and $M_{2}$ on a sample, the curvatures of the largest face along the $x$ and $y$ axis, i.e. the principal curvatures in the two perpendicular directions $x$ and $y$, turn out to be

$$
\frac{1}{R_{x}}=\frac{\mathrm{d}^{2} w}{\mathrm{~d} x^{2}}=-\left(\frac{M_{1}}{I_{x}} S_{11}+\frac{M_{2}}{I_{y}} S_{12}\right)
$$

and

$$
\frac{1}{R_{y}}=\frac{\mathrm{d}^{2} w}{\mathrm{~d} y^{2}}=-\left(\frac{M_{1}}{I_{x}} S_{12}+\frac{M_{2}}{I_{y}} S_{22}\right) .
$$

Through equations (5) and (6), all deformations of the largest surface are calculable. As an example, if we apply only $M_{1}$ or only $M_{2}$ on plate, the anticlastic ratio $\left(R_{x} / R_{y}\right)$ can be calculated as

$$
\begin{aligned}
& \frac{R_{x}}{R_{y}}=\frac{S_{12}}{S_{11}} \quad \text { if } \quad M_{2}=0, \\
& \frac{R_{y}}{R_{x}}=\frac{S_{12}}{S_{22}} \quad \text { if } \quad M_{1}=0 .
\end{aligned}
$$

\section{QM curvature calculation}

A crystalline material may present nondiagonal components in the compliance tensor. Thus, nontrivial deformation can arise as a consequence of external forces. A crystal plate, subjected to two mechanical moments $M_{1}$ and $M_{2}$ applied around the $y$ and $x$ axis (see Fig. 2a), undergoes a primary deformation. The secondary curvatures depend on crystal anisotropy. Thus, their radii of curvature are strictly linked to the crystal orientation. All crystal deformations can be calculated through the displacement field by using equation (3). In particular, the ratio of the primary and secondary curvatures can be calculated. The QM curvature in the $x z$. plane is

$$
\frac{1}{R_{\mathrm{QM}}}=\frac{\mathrm{d}^{2} u}{\mathrm{~d} z^{2}}=\frac{M_{1}}{I_{x}} S_{51}+\frac{M_{2}}{I_{y}} S_{52},
$$

while that in the $y z$ plane is

$$
\frac{1}{R_{\mathrm{QM}}}=\frac{\mathrm{d}^{2} v}{\mathrm{~d} z^{2}}=\frac{M_{1}}{I_{x}} S_{41}+\frac{M_{2}}{I_{y}} S_{42} .
$$

The results shown in equations (9) and (10) hold true for any kind of crystals. In order to have the QM curvature, at least one term among $S_{41}, S_{42}, S_{51}$ or $S_{52}$ must not be zero. In particular, to obtain QM curvature in the $y z$ plane, $S_{41}$ or $S_{42}$ must not be zero. In the same way, the QM effect in the $x z$ plane occurs only if one of $S_{51}$ or $S_{52}$ is not zero. Actually, such terms of the compliance tensor are always zero for the crystallographic orientation normally used in applications.

Here, we report three examples for $\mathrm{Si}$ and Ge crystals, which are the most used crystals for a wide range of applications and experiments. In the examples, the crystal plates are deformed by one applied moment $\left(M_{1} \neq 0, M_{2}=0\right.$ or $\left.M_{1}=0, M_{2} \neq 0\right)$ or by two identical applied moments $\left(M_{1}=M_{2} \neq 0\right)$. These are the most common cases of deformation. As explained in $\S 6$, a curvature due to one moment can be obtained, for example, by using an external holder (Carassiti et al., 2010) or by applying the grooving method (Bellucci, Camattari, Guidi \& Mazzolari, 2011; Camattari, Guidi, Lanzoni \& Neri, 2013). This latter method can also be used to apply two identical moments to a plate, which can alternatively be done by depositing a tensile or compressive film (Guidi Lanzoni \& Mazzolari, 2011; Camattari, Dolcini et al., 2014; Mazzolari et al., 2015) or through surface damage (Ferrari et al., 2013). In $\S 7$ of this paper, a more general case is treated in which the two applied moments are not equal to each other $\left(M_{1} \neq 0, M_{2} \neq 0, M_{1} \neq M_{2}\right)$.

We consider the three configurations described in Figs. 2(b)-2(d).

\subsection{QM curvature in Si and Ge crystals: case (a)}

For the first case (Fig. 2b), the compliance tensors for Si and $\mathrm{Ge}$ at room temperature are given by

$$
\begin{aligned}
S_{\mathrm{Si}} & =\left(\begin{array}{cccccc}
0.592 & -0.096 & -0.155 & 0.167 & 0 & 0 \\
-0.096 & 0.533 & -0.096 & 0 & 0 & 0 \\
-0.155 & -0.096 & 0.592 & -0.167 & 0 & 0 \\
0.167 & 0 & -0.167 & 1.730 & 0 & 0 \\
0 & 0 & 0 & 0 & 1.494 & 0.334 \\
0 & 0 & 0 & 0 & 0.334 & 1.730
\end{array}\right) \\
S_{\mathrm{Ge}} & =\left(\begin{array}{cccccc}
0.726 & -0.101 & -0.181 & 0.228 & 0 & 0 \\
-0.101 & 0.646 & -0.101 & 0 & 0 & 0 \\
-0.181 & -0.101 & 0.726 & -0.228 & 0 & 0 \\
0.228 & 0 & -0.228 & 2.138 & 0 & 0 \\
0 & 0 & 0 & 0 & 1.815 & 0.456 \\
0 & 0 & 0 & 0 & 0.456 & 2.138
\end{array}\right)
\end{aligned}
$$

In this case, $S_{51}$ and $S_{52}$ are both equal to zero. Thus, the QM curvature can be obtained only in the $y z$ plane, i.e. it concerns the (111) planes. Considering a curvature under the condition $M_{1} / I_{x}=M_{2} / I_{y}$ (uniform distribution of stress), the ratio of the primary and QM curvature is

$$
\frac{R_{\mathrm{QM}}}{R_{x}}=-\frac{S_{11}+S_{12}}{S_{41}+S_{42}}, \quad \frac{R_{\mathrm{QM}}}{R_{y}}=-\frac{S_{12}+S_{22}}{S_{41}+S_{42}} .
$$

In the case of a single moment applied, the ratio is 


$$
\begin{array}{lll}
\frac{R_{\mathrm{QM}}}{R_{x}}=-\frac{S_{11}}{S_{41}} \quad \text { if } & M_{2}=0, \\
\frac{R_{\mathrm{QM}}}{R_{y}}=-\frac{S_{22}}{S_{42}} \quad \text { if } & M_{1}=0 .
\end{array}
$$

From equations (11) and (12), it can be noticed that $S_{42}=0$. Thereby, if $M_{1}=0$, no QM deformation occurs. In contrast, if at least $M_{1} \neq 0, \mathrm{QM}$ curvature appears in the $y z$ plane, i.e. the (111) planes are bent owing to 'quasi-mosaicity'. X-ray diffraction by (111) planes bent through the QM effect was observed experimentally for $\mathrm{Si}$ and Ge crystals by Camattari, Guidi, Bellucci et al. (2013) and Camattari, Paternò, Battelli et al. (2014), respectively. An example of the use of (111) planes bent by the QM effect for particle steering is given by Scandale, Vomiero, Bagli et al. (2009).

\subsection{QM curvature in Si and Ge crystals: case (b)}

For the second case (Fig. 2c), the compliance tensors for $\mathrm{Si}$ and $\mathrm{Ge}$ at room temperature are

$$
\begin{aligned}
S_{\mathrm{Si}} & =\left(\begin{array}{cccccc}
0.592 & -0.155 & -0.096 & 0.167 & 0 & 0 \\
-0.155 & 0.592 & -0.096 & -0.167 & 0 & 0 \\
-0.096 & -0.096 & 0.533 & 0 & 0 & 0 \\
0.167 & -0.167 & 0 & 1.73 & 0 & 0 \\
0 & 0 & 0 & 0 & 1.73 & 0.334 \\
0 & 0 & 0 & 0 & 0.334 & 1.494
\end{array}\right) \\
S_{\mathrm{Ge}} & =\left(\begin{array}{cccccc}
0.726 & -0.181 & -0.101 & 0.228 & 0 & 0 \\
-0.181 & 0.726 & -0.101 & -0.228 & 0 & 0 \\
-0.101 & -0.101 & 0.646 & 0 & 0 & 0 \\
0.228 & -0.228 & 0 & 2.138 & 0 & 0 \\
0 & 0 & 0 & 0 & 2.138 & 0.456 \\
0 & 0 & 0 & 0 & 0.456 & 1.815
\end{array}\right)
\end{aligned}
$$

Also in this case, $S_{51}$ and $S_{52}$ are both zero; thus, the QM curvature can be obtained only in the $y z$ plane, i.e. it involves

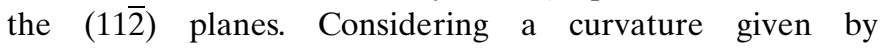
$M_{1} / I_{x}=M_{2} / I_{y}$, the ratio of the primary and QM curvature is

$$
\frac{R_{\mathrm{QM}}}{R_{x}}=-\frac{S_{11}+S_{12}}{S_{41}+S_{42}}, \quad \frac{R_{\mathrm{QM}}}{R_{y}}=-\frac{S_{12}+S_{22}}{S_{41}+S_{42}} .
$$

However, in this case, $S_{41}+S_{42}=0$. Thus, a deformation due to two identical moments would cause the disappearance of the QM curvature. Conversely, a single moment can be applied on the plate for obtaining the primary curvature, giving rise to QM curvature in the (11/2) planes. If $M_{1} \neq 0$ and $M_{2}=0$, the primary curvature along the $x$ axis is

$$
\frac{1}{R_{x}}=-\frac{M_{1}}{I_{x}} S_{11},
$$

while the secondary curvature in the $y z$ plane is

$$
\frac{1}{R_{\mathrm{QM}}}=\frac{M_{1}}{I_{x}} S_{42} .
$$

The ratio of the QM and primary curvature can be obtained as

$$
\frac{R_{\mathrm{QM}}}{R_{x}}=-\frac{S_{11}}{S_{42}} .
$$

If $M_{1}=0$ and $M_{2} \neq 0$, the primary curvature along the $y$ axis is

$$
\frac{1}{R_{y}}=-\frac{M_{2}}{I_{y}} S_{22}
$$

The ratio of the QM and primary curvature in this case is

$$
\frac{R_{\mathrm{QM}}}{R_{y}}=-\frac{S_{22}}{S_{42}} .
$$

Experimental evidence of X-ray diffraction by an Si sample obtained by exploiting (112) planes bent by the QM effect is given by Bellucci et al. (2013).

\subsection{QM curvature in Si and Ge crystals: case (c)}

The compliance tensors for $\mathrm{Si}$ and $\mathrm{Ge}$ at room temperature for the case depicted in Fig. 2(d) are

$$
S_{\mathrm{Si}}=\left(\begin{array}{cccccc}
0.631 & -0.178 & -0.112 & -0.103 & 0.117 & -0.11 \\
-0.178 & 0.658 & -0.139 & 0.190 & 0.026 & 0.057 \\
-0.112 & -0.139 & 0.592 & -0.087 & -0.142 & 0.053 \\
-0.103 & 0.19 & -0.087 & 1.558 & 0.105 & 0.052 \\
0.117 & 0.026 & -0.142 & 0.105 & 1.666 & -0.206 \\
-0.110 & 0.057 & 0.053 & 0.052 & -0.206 & 1.402
\end{array}\right)
$$

$$
S_{\mathrm{Ge}}=\left(\begin{array}{cccccc}
0.780 & -0.213 & -0.123 & -0.141 & 0.159 & -0.150 \\
-0.213 & 0.816 & -0.159 & 0.260 & 0.035 & 0.078 \\
-0.123 & -0.159 & 0.726 & -0.119 & -0.194 & 0.072 \\
-0.141 & 0.26 & -0.119 & 1.903 & 0.144 & 0.071 \\
0.159 & 0.035 & -0.194 & 0.144 & 2.05 & -0.281 \\
-0.150 & 0.078 & 0.072 & 0.071 & -0.281 & 1.69
\end{array}\right)
$$

In this case, it is possible to obtain the QM effect for both the planes perpendicular to the $x$ and $y$ axes because $S_{41}, S_{42}, S_{51}$ and $S_{52}$ are not zero. Following the same procedure reported above, considering a curvature given by $M_{1} / I_{x}=M_{2} / I_{y}$, the ratios of the primary and QM curvature of the (174) planes are

$$
\frac{R_{\mathrm{QM}}}{R_{x}}=-\frac{S_{11}+S_{12}}{S_{51}+S_{52}}, \quad \frac{R_{\mathrm{QM}}}{R_{y}}=-\frac{S_{12}+S_{22}}{S_{51}+S_{52}},
$$

while the QM curvature along the (311) planes is

$$
\frac{R_{\mathrm{QM}}}{R_{x}}=-\frac{S_{11}+S_{12}}{S_{41}+S_{42}}, \quad \frac{R_{\mathrm{QM}}}{R_{y}}=-\frac{S_{12}+S_{22}}{S_{41}+S_{42}} .
$$

In the case of a single moment, the QM curvature of the (174) planes is related to the primary curvature as

$$
\begin{aligned}
& \frac{R_{\mathrm{QM}}}{R_{x}}=-\frac{S_{11}}{S_{51}}, \quad \frac{R_{\mathrm{QM}}}{R_{y}}=-\frac{S_{12}}{S_{51}} \quad \text { if } \quad M_{2}=0, \\
& \frac{R_{\mathrm{QM}}}{R_{x}}=-\frac{S_{12}}{S_{52}}, \quad \frac{R_{\mathrm{QM}}}{R_{y}}=-\frac{S_{22}}{S_{52}} \quad \text { if } \quad M_{1}=0 .
\end{aligned}
$$


Table 1

Ratios of the QM and principal curvature in a deformed crystal with $M_{1} / I_{x}=M_{2} / I_{y}$.

\begin{tabular}{llllll}
\hline Case & Material & $R_{\mathrm{QM}_{x}} / R_{x}$ & $R_{\mathrm{QM}_{x}} / R_{y}$ & $R_{\mathrm{QM}_{y}} / R_{x}$ & $R_{\mathrm{QM}_{y}} / R_{y}$ \\
\hline$a$ & $\mathrm{Si}$ & $\infty$ & $\infty$ & 2.97 & 2.61 \\
$a$ & $\mathrm{Ge}$ & $\infty$ & $\infty$ & 2.74 & 2.39 \\
$b$ & $\mathrm{Si}$ & $\infty$ & $\infty$ & $\infty$ & $\infty$ \\
$b$ & $\mathrm{Ge}$ & $\infty$ & $\infty$ & $\infty$ & $\infty$ \\
$c$ & $\mathrm{Si}$ & -3.18 & -3.37 & -5.19 & -5.50 \\
$c$ & $\mathrm{Ge}$ & -2.92 & -3.10 & -4.76 & -5.07 \\
\hline
\end{tabular}

In the case of a single moment, the QM curvature of the (311) planes is related to the primary curvature as

$$
\begin{array}{ll}
\frac{R_{\mathrm{QM}}}{R_{x}}=-\frac{S_{11}}{S_{41}}, & \frac{R_{\mathrm{QM}}}{R_{y}}=-\frac{S_{12}}{S_{41}} \quad \text { if } \quad M_{2}=0, \\
\frac{R_{\mathrm{QM}}}{R_{x}}=-\frac{S_{12}}{S_{42}}, & \frac{R_{\mathrm{QM}}}{R_{y}}=-\frac{S_{22}}{S_{42}} \quad \text { if } \quad M_{1}=0 .
\end{array}
$$

In spite of the relatively high Miller indices, the diffraction efficiency of such planes is not negligible. Experimental evidence for this observation in the case of an $\mathrm{Si}$ crystal is given by Camattari, Paternò, Bellucci \& Guidi (2014).

The ratios for the cases described above are reported in Tables 1 and 2 .

\section{Techniques to fabricate QM crystals}

Since the QM effect is a consequence of crystal anisotropy, it does not depend on the method used for manufacturing the sample. However, the best technique to fabricate a QM crystal with the desired curvature depends on the application.

The simplest technique to fabricate a QM crystal consists of using an external holder capable of imparting a single mechanical moment to a crystal plate, or in some particular cases, two perpendicular moments. The holder is typically made of duralumin or titanium; the latter is preferable if the holder has to be baked for ultra-high vacuum. The holder performs two tasks: it holds the sample and applies the mechanical moments on the sample for bending.

Holders were used in all experiments on charged-particle steering through channeling and volume reflection reported in this paper. As an example, we show in Fig. 3 the holder used for bending a QM Si crystal in an experiment performed at the Mainzer Mikrotron (MAMI) (Mazzolari et al., 2014).

An external holder cannot be used to bend a crystal for the applications of $\mathrm{X}$ - and $\gamma$-ray focusing. Observations of the sky in this energy range must necessarily be performed in satelliteborne experiments to avoid absorption by the atmosphere. For any space mission, the payload is a crucial parameter dictating that the curved crystal must be self-standing. In nuclear medicine, there are no weight constraints; however, the need for the miniaturization of optical components requires that no assistance be given by mechanical devices. Fabrication of selfstanding crystals should be compatible with mass production techniques, especially when hundreds or thousands of elements have to be manufactured, for example for astro-
Table 2

Ratios of QM and principal curvature in a crystal deformed by a single

\begin{tabular}{|c|c|c|c|c|c|}
\hline Case & Material & $\frac{M_{2}=0}{R_{\mathrm{QM}_{x}} / R_{x}}$ & $\frac{M_{2}=0}{R_{\mathrm{QM}_{y}} / R_{x}}$ & $\frac{M_{1}=0}{R_{\mathrm{QM}_{x}} / R_{y}}$ & $\frac{M_{1}=0}{R_{\mathrm{QM}_{y}} / R_{y}}$ \\
\hline$a$ & $\mathrm{Si}$ & $\infty$ & -3.54 & $\infty$ & $\infty$ \\
\hline$a$ & $\mathrm{Ge}$ & $\infty$ & -3.18 & $\infty$ & $\infty$ \\
\hline$b$ & $\mathrm{Si}$ & $\infty$ & -3.54 & $\infty$ & 3.54 \\
\hline$b$ & $\mathrm{Ge}$ & $\infty$ & -3.18 & $\infty$ & 3.18 \\
\hline$c$ & $\mathrm{Si}$ & -5.41 & 6.12 & -25.39 & -3.46 \\
\hline$c$ & $\mathrm{Ge}$ & -4.90 & 5.54 & -23.08 & -3.14 \\
\hline
\end{tabular}
moment.

physical applications. A technique that satisfies the requirements of low cost and reproducibility is the grooving method, which was proposed by Bellucci et al. (2003) and extensively developed later by Camattari, Guidi, Lanzoni \& Neri (2013).

The grooving method consists of creating a series of scratches on one of the major surfaces of a plate. It was shown that a series of superficial grooves may permanently and reproducibly bend the whole crystal owing to the generation of a plasticized layer in the neighborhood of the grooves. If a set of parallel grooves is made on a crystal, a single moment is imparted to the plate. If a grid of grooves is created, two identical moments are transferred to the crystal. We show in Fig. 4, as an example, some grooved samples produced within the Laue project for the realization of a Laue lens prototype (Camattari, Battelli et al., 2013). The samples are $10 \times 30 \times$ $2 \mathrm{~mm}$ Ge crystals, oriented as in Fig. 2(b).

However, with the grooving method, it is not possible to bend plates thicker than $2 \mathrm{~mm}$ to the desired curvature for an application. Thicker self-standing bent crystals were recently obtained by the deposition of a thick film composed of carbon fiber. Crystals of up to $5 \mathrm{~mm}$ thickness were bent with the radius of primary curvature down to $30 \mathrm{~m}$ (Camattari, Dolcini et al., 2014; Mazzolari et al., 2015). Moreover, this technique offers the advantage of not damaging the substrate. A

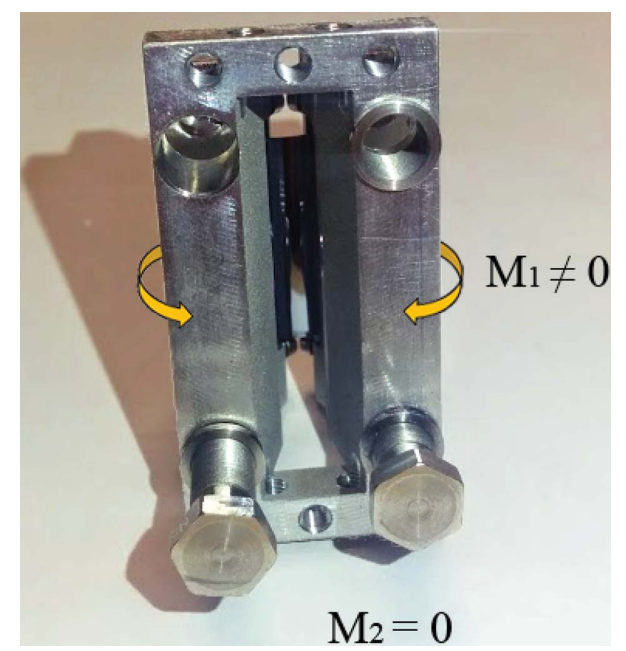

Figure 3

Photograph of the Si QM sample. The holder is made of ergal. The screws at the bottom are used to adjust the sample curvature. The holder is $36 \times$ $50 \mathrm{~mm}$ in area and $17 \mathrm{~mm}$ thick, while the Si QM sample is $15 \times 23 \mathrm{~mm}$ in area and $30.5 \mu \mathrm{m}$ thick. Here $M_{1} \neq 0, M_{2}=0$. 
photograph of a QM Si sample bent by the deposition of a carbon fiber film is shown in Fig. 5.

One important aspect in the manufacturing of a QM crystal is the reproducibility of the curvature due to 'quasi-mosaicity', i.e. the spread of the QM curvature and the alignment of the QM planes with respect to the crystal plate. Since the QM curvature strictly depends on the primary curvature, the first requirement for a reproducible QM curvature is a reproducible primary curvature. However, even if the QM crystals are produced using the same technique and have the same primary curvature, uncertainties in the crystallographic orientation of the crystal plates may result in different QM curvatures. This aspect may become critical in experiments where a large number of identical crystals are required, such as for fabrication of a Laue lens. Typical errors during the crystal manufacturing are the misalignment between crystal surfaces and crystallographic planes. These errors are named miscut and misflat errors and can be of the order of $\pm 0.1^{\circ}$. Taking into account these uncertainties, the influence on the QM curvature is very poor, less than $1 \%$. As a practical example, let us consider an Si or Ge crystal with a spherical curvature of $40 \mathrm{~m}$, oriented as in Fig. 2(b). An uncertainty of $\pm 0.1^{\circ}$ on both miscut and misflat angles produces an uncertainty on the angular spread of the QM planes of 0.9 and $0.6 \%$ for $\mathrm{Si}$ and $\mathrm{Ge}$, respectively. These calculations have been performed through a ready-to-use software, named AniCryDe (Camattari et al., 2015).

In the following two sections, we provide two recent applications of 'quasi-mosaicity' in modern physics. The first is charged-particle steering for accelerator technology and highenergy physics (\$5); the second is X- and $\gamma$-ray diffraction for astrophysics and nuclear medicine applications (§6).

\section{QM crystals for steering charged particles}

Charged particles traversing an amorphous material or a misaligned crystal undergo uncorrelated collisions with the atoms of the medium. During the interaction, particles are

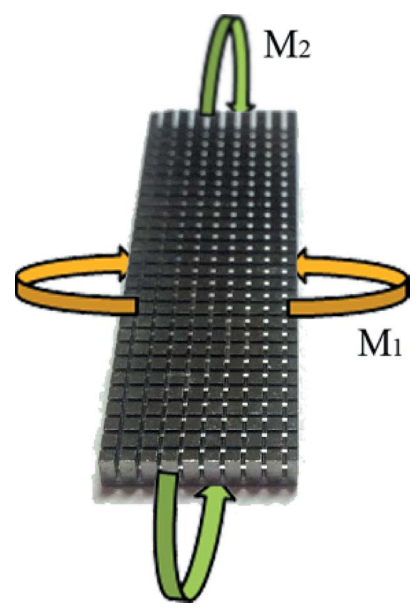

Figure 4

Photograph of a Ge QM sample manufactured as an X-ray optical element. The sample is $10 \times 30 \times 2 \mathrm{~mm}$ in size. The grid of grooves is clearly visible. Here $M_{1}=M_{2} \neq 0$. randomly scattered because of incoherent interactions with atomic nuclei, and energy loss occurs because of collisions with atomic electrons. In 1964, Lindhard introduced the concept of coherent interactions of charged particles with trajectories nearly parallel to a crystal plane or axis (Lindhard, 1965). In this case, correlated interactions occur between all atoms in a string or plane. On the basis of the concept of coherent interactions, Lindhard provided an explanation about the phenomenology of channeling. Channeling occurs as a charged-particle beam impinges onto a crystal at an angle less than the critical angle with respect to a crystal plane (axis). The motion of channeled particles gets confined between neighboring planes (axes) that become preferential pathways for the trajectory of the particles inside the crystal.

Initially, channeling was identified as an unwanted sideeffect in ion implantation doping at the dawn of the semiconductor era (Piercy et al., 1963). Indeed, if an ion beam is aligned with a crystal at an angle less than the critical angle, ions would penetrate too deeply into the bulk because of channeling. Later, scientists exploited the channeling of lowenergy particle beams $\left(\mathrm{p}^{+}\right.$and $\mathrm{He}^{++}$in the $\mathrm{MeV}$ energy range) to perform material analysis and submicrometre crystallography for the characterization of crystal defects (Feldman et al., 1982). Another application of channeling was the study of radiation emission by channeled electrons or positrons, which oscillate during their motion inside a crystal (Kumakhov, 1976). This phenomenon was called channeling radiation and has been exploited for the production of intense electromagnetic radiation (Lohmann et al., 1994).

Since 1976, owing to a seminal idea by E. N. Tsyganov (Tsyganov, 1976a; Elishev et al., 1979), channeling in appropriately bent crystals has been proposed for steering of charged-particle beams. Channeled particles follow the crystal curvature, resulting in a deflection by an angle equal to the crystal bending angle (see Fig. $6 a$ ). In principle, a bent crystal can be used to perform all operations on channeled particles that are normally accomplished by magnets in particle accelerators, such as deflection, splitting, undulation, extraction and collimation. It took more than 20 years to sufficiently develop

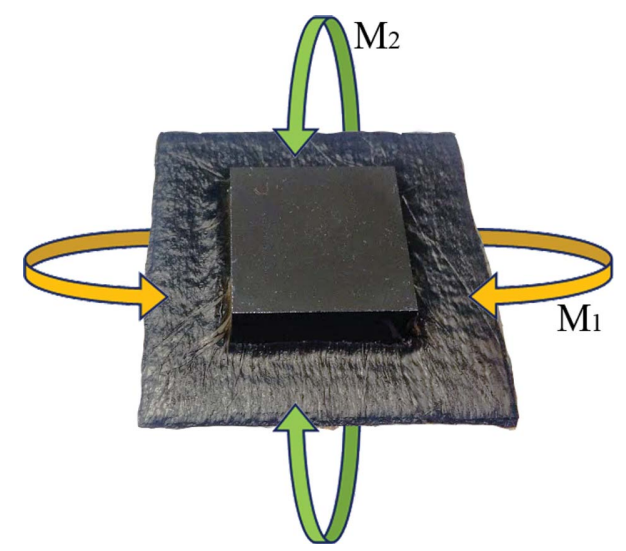

Figure 5

Photograph of a QM Si sample bent by deposition of a carbon fiber film. Here $M_{1}=M_{2} \neq 0$. The sample is $20 \times 20 \mathrm{~mm}$ in area and $5 \mathrm{~mm}$ thick; the carbon fiber film is $\sim 40 \times 40 \times 0.6 \mathrm{~mm}$ in size. 
the technology to fabricate crystals that could enable chargedparticle beam manipulation with high efficiency (Biryukov et al., 2002).

The first successful experiment on the high-efficiency manipulation of a proton beam was carried out by bending a strip-like Si crystal through anticlastic deformation (Afonin et al., 2001). Later, the same results were achieved with QM crystals (Ivanov et al., 2006). In contrast to the scheme based on anticlastic deformation (Guidi et al., 2010), a QM crystal is positioned perpendicularly to the particle beam, as shown in Fig. 6(b).

The UA9 collaboration installed a QM crystal and a crystal relying on anticlastic deformation as candidates for collimating the $7 \mathrm{TeV}$ proton beam in the LHC (Scandale et al., 2011). Actually, halo collimation in the LHC is mandatory to achieve the top luminosity of the machine and to prevent any damage to superconductor magnets. The problem of collimation is not trivial, because the halo intensity in the LHC is comparable to the intensity of the full beam in the CERN SPS. The crystal would act as a primary collimator, steering the particles in the halo towards a massive absorber. Preliminary collimation tests were performed on the SPS circular accelerator, resulting in a strong reduction in beam losses in the case of both protons (Scandale et al., 2010) and $\mathrm{Pb}$ ions (Scandale et al., 2008a). On-beam tests of collimation in the LHC are being planned for 2015.

A QM crystal highlights two interesting features for channeling experiments. First, it allows the interception of a broad beam because the largest face of the crystal is exposed to the beam. Second, the thickness of a QM crystal along the beam direction can be adjusted to a few tens of micrometres, which is a value not achievable in the case of traditional bent crystals.

To maximize deflection efficiency, it is important to design crystals with optimized characteristics, e.g. the crystal length along the beam direction. An important parameter to quantify the channeling efficiency is the dechanneling length; this is the

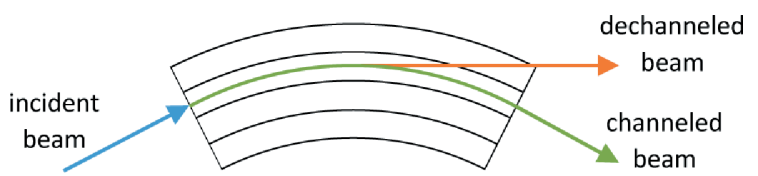

(a)

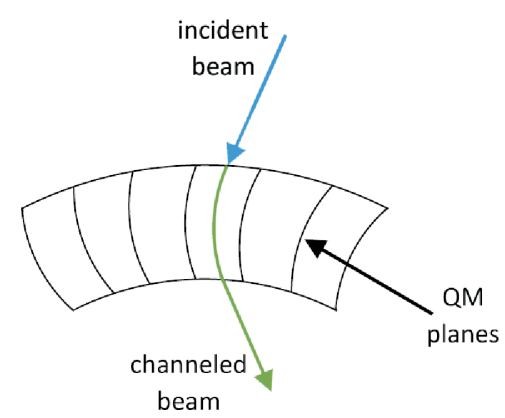

Figure 6

(a) Schematic representation of channeling. An incident charged-particle beam entering a crystal at an angle less than the critical angle can be deflected along the crystal curvature. (b) Schematic representation of a QM crystal used for steering a charged-particle beam. average length within which a channeled particle leaves the channeling condition with a probability of $(1-1 / e)$ (Biryukov et al., 1997). To efficiently steer a charged-particle beam, the crystal thickness must not be higher than the dechanneling length, which depends on beam energy. For instance, the use of QM crystals allowed the deflection of GeV-energy charged particles by means of a crystal with a thickness of less than one millimetre (Ivanov et al., 2006).

QM crystals allowed the first experimental observation of a phenomenon that had been predicted for many years, namely volume reflection (VR) (Taratin \& Vorobiev, 1987). VR occurs as an over-barrier particle traveling through the crystal arrives at the tangential point with the bent crystalline planes. At this point, elastic reflection of the transverse component of the momentum takes place, resulting in a net deflection of the particle trajectory towards the side opposite to that of the crystal curvature (see Fig. 7). VR occurs with extremely high efficiency, though its angular deflection is smaller than that for channeling. VR was experimentally observed at the CERN SPS H8 external line in the case of a $400 \mathrm{GeV}$ proton beam with $97 \%$ efficiency (Scandale et al., 2008b).

As can be seen in Fig. 7, most of a crystal is traversed by a particle as if it was an amorphous medium. Coherent interaction plays a dominant role in the neighborhood of the tangency point, where the transverse component of the particle momentum is reversed. Apart from this region, the particle interacts incoherently with the atoms in the crystal, resulting in a smearing out of the beam. Since the deflection due to VR is small, the effect of incoherent interactions may hide the effect of VR. In principle, such a portion of the crystal could be removed, leaving a crystal purely working under the VR regime. Such a crystal, which is yet to be realized, could only be used via the QM effect. As an example, a crystal designed to work with $400 \mathrm{GeV}$ protons would have a thickness of a few tens of micrometres, which is indeed achievable with current technology involving 'quasi-mosaicity'.

The property of QM crystals to be thinned to a very low thickness can be even more important when the channeling of negatively charged particles is concerned. The dechanneling length of negative particles is far shorter than that of positive particles. Indeed, negative particles repeatedly oscillate across crystallographic planes/axes (Scandale et al., 2013). Thus, the probability of inelastic scattering of channeled negative particles by atomic electrons, and especially the probability of multiple scattering by lattice atoms, is much higher than that in the case of positive particles. The use of a QM crystal for steering negative particles was proposed by Guidi et al. (2009). Then, QM crystals were effectively used for this purpose in

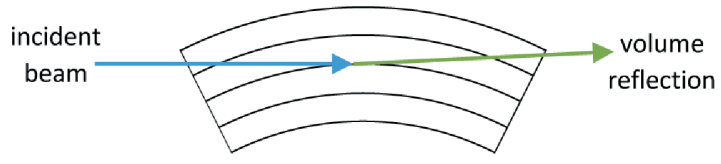

Figure 7

Schematic representation of volume reflection. A charged-particle beam can be deflected towards the opposite side with respect to the crystal curvature. 
two experiments, at CERN (Scandale, Vomiero, Bagli et al., 2009) and at MAMI (Mazzolari et al., 2014). In these experiments, both channeling and VR were observed.

QM crystals can also be used to produce electromagnetic radiation as follows. It is known that a charged particle traversing a medium loses kinetic energy, which is partly converted into electromagnetic radiation (the bremsstrahlung effect; Bethe, 1934). Photon emission through coherent bremsstrahlung is more intense than for the incoherent case (Baier et al., 1998). Moreover, if the crystal is bent, it is possible to conveniently exploit VR to produce radiation. Such radiation is as intense as in the channeling case, but it exhibits larger angular acceptance of the incident particles and circumvents the problem of dechanneling (Scandale, Vomiero, Baricordi et al., 2009; Bandiera et al., 2014). Lighter particles generate the highest intensity radiation because bremsstrahlung strongly decreases with particle mass. Since there exist worldwide a number of electron accelerators delivering $\mathrm{GeV}$ or sub-GeV beams, the study of channeling radiation emitted by light particles at these energies is of particular interest in view of possible applications such as a crystalline undulator (Tabrizi et al., 2007; Bagli et al., 2014).

\section{QM crystals for focusing hard X-rays}

Manipulation and focusing of hard X-and $\gamma$-rays in the 100 $1000 \mathrm{keV}$ energy range represents an increasingly significant

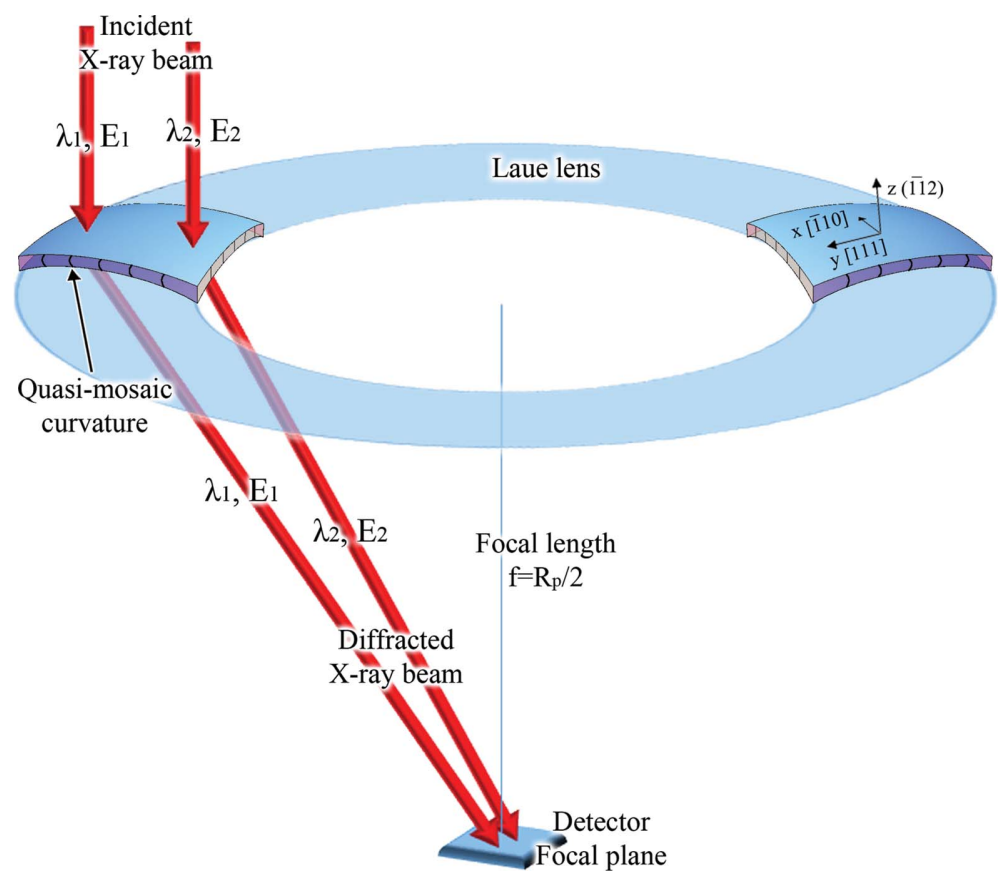

Figure 8

Schematic representation of a Laue lens based on QM crystals. Left side: red arrows represent an X-ray beam diffracted towards a detector placed on the focal plane of the lens. The blue plate represents the crystal. The primary curvature leads to a secondary curvature of the planes affected by 'quasi-mosaicity'. In this configuration, QM diffracting planes are orthogonal to the main surface of the plate. The primary curvature allows focusing of the diffracted radiation onto the focal plane, while the QM curvature increases the integrated diffraction efficiency. Right side: the particular arrangement of a QM crystal in a Laue lens. topic for the scientific community. However, it is not easy to focalize X-rays with good efficiency, and the modalities of the implementation of an X-ray concentrator still represent an open issue.

Nowadays, the study of hard X-ray or soft $\gamma$-ray astronomical sources in the $100-1000 \mathrm{keV}$ energy range is carried out by observing the sky without the help of telescopes because a high-energy photon beam cannot be concentrated. Indeed, the lack of optical components capable of working within this energy range implies the impossibility of focusing, which in turn leads to a poor signal-to-noise ratio recorded by the detectors. Multilayer optics have been proved to focus up to $80 \mathrm{keV}$ photons with high efficiency. Very recently, it has been demonstrated that multilayer reflective optical components can operate efficiently up to photon energies of at least $600 \mathrm{keV}$ (Fernández-Perea et al., 2013; Brejnholt et al., 2014). However, these new reflective optics work at very low grazing incidence angles (below $0.1^{\circ}$ ). This means that they have a very low acceptance area for the incident photons, and beyond these energy limits, their efficiency critically deteriorates.

If X-ray diffraction occurs in Laue geometry, namely if photons traverse a crystal, hard X-rays can be focused via a Laue lens (Lund, 1992). A Laue lens is conceived as an ensemble of many crystals arranged in such a way that the maximum possible radiation is diffracted towards the lens focus over a selected energy band.

In order to effectively detect X-rays by using a Laue lens, optical elements with high diffraction efficiency are needed, along with an arrangement of crystals to permit high-resolution focusing of the diffracted photons.

In order to increase integrated diffraction efficiency, the scientific community has selected mosaic and curved crystals (Authier \& Malgrange, 1998). The use of bent crystals represents an elegant solution because they can diffract radiation with very high efficiency and with a controlled and uniform energy passband (Bellucci, Camattari, Guidi, Neri \& Barrière, 2011). In the framework of bent crystals, QM crystals offer further opportunities for building a Laue lens. Indeed, it is possible to exploit 'quasimosaicity' to obtain high-resolution focusing of diffracted photons (Camattari et al., 2011).

A Laue lens based on QM crystals consists of an arrangement of curved crystals whose primary curvature lies on a spherical calotte of radius $R$, while the QM curvature allows diffraction by curved diffracting planes. The use of QM crystals allows positioning of crystals in a Laue lens in the same way as for mosaic crystals, i.e. with the diffracting planes orthogonal to the major faces of the crystal (Fig. 8). For a Laue lens made of crystals with diffracting planes orthogonal to the major face of the crystal, focusing can be fully provided by bending the crystals to a primary curvature equal to that of the whole lens. Owing to Bragg diffraction, focusing of each QM sample converges on a focal spot at a distance $f=R / 2$ 
on the symmetry axes of the calotte (see Fig. 8). By using QM crystals, it is possible to encompass the focusing action due to the primary curvature with the high reflectivity of bent diffracting planes provided by 'quasi-mosaicity'. In fact, if there were no QM effect, the integrated reflectivity would be equal to that in the case of a crystal with flat diffracting planes, which is a relatively poor figure. Since the size of the focal spot of the photons diffracted by a QM crystal can be controlled by adjusting the primary curvature, QM crystals allow focusing with higher resolution than that achievable using mosaic or traditional curved crystals. In particular, 'quasi-mosaicity' allows focusing of a photon flux in a spot smaller than the size of the diffracting crystal through bent diffracting planes, i.e. with high integrated diffraction efficiency. This is in contrast to diffraction by a flat mosaic crystal, the spot of which is no smaller than the crystal size exposed to the photons. As a result, the sensitivity of a Laue lens would be significantly increased.

QM crystals have been employed for realizing a Laue lens prototype with a focal length of $20 \mathrm{~m}$, as part of the Laue project which is financed by the Italian Space Agency (ASI) (Virgilli et al., 2011). The samples were Ge crystals oriented as shown in Fig. 2(b). The sample size was $10 \times 30 \times 2 \mathrm{~mm}$ (Camattari, Battelli et al., 2013). The prototype Laue lens based on QM crystals is under development at the LARIX facility (Loffredo et al., 2005) (Ferrara, Italy) as part of the Laue project (Frontera et al., 2012). The focal spot on a detector placed at the focal distance of a Laue lens based on such crystals can be very small, of the order of a few millimetres (Bellucci et al., 2013; Camattari, Paternò, Bellucci \& Guidi, 2014).

\section{Adjustable QM curvature}

It has been demonstrated that the curvature of the crystallographic planes due to 'quasi-mosaicity' may be very useful for some applications. In any case, the QM curvature is lower than the primary curvatures because the ratio of the primary and $\mathrm{QM}$ curvature is always greater than $\sim 2.4$. However, if it was possible to increase the QM curvature, some improvements would be achieved. Firstly, for channeling experiments, a crystal with a larger QM curvature would result in stronger deflection. Secondly, for VR experiments, a larger QM curvature would guarantee broader angular acceptance of the incident beam. Finally, for diffraction experiments, a crystal with a larger QM curvature would imply higher integrated diffraction efficiency.

The crystals fabricated to exploit the QM effect have always been bent by applying either a single moment or two identical moments $\left(M_{1}=M_{2}\right)$. In this work, we demonstrate that the QM curvature may be increased, provided that two nonvanishing moments with different intensities are applied on the crystal plate, i.e. $M_{1} \neq M_{2} \neq 0$.

As an example, let us consider a Laue lens entirely relying on QM crystals, with the samples oriented as shown in Fig. 2(b). As explained in $\S 6$, a QM crystal focuses the diffracted photons to a focal spot smaller than the tile size (see
Fig. 8). Consider a crystal arranged as shown on the right side of Fig. 8, with the $y$ axis of the plate directed along the radial direction of the Laue lens. For focusing, the relation $R_{y}=2 f$ must hold true, where $f$ is the focal length of the lens. Thus, this radius of curvature is frozen. The radius of curvature in the direction perpendicular to the radial direction, $R_{x}$, is a free parameter. By decreasing $R_{x}$, it would be possible to increase the QM curvature, and therefore to enhance the lens performance, while keeping fixed the geometry of the lens.

More quantitatively, the value of the QM curvature can be calculated by taking into account different values for $M_{1}$ and $M_{2}$ by applying equations (5)-(10). Fig. 9 shows the QM radius of curvature, $R_{\mathrm{OM}}$, as a function of the radius of curvature along the $x$ axis $\left(R_{x}\right)$ and $y$ axis $\left(R_{y}\right)$. As can be seen in the figure, by increasing the curvature along the $x$ axis, i.e. by decreasing $R_{x}, R_{\mathrm{QM}}$ decreases too.

Since it is possible to increase the QM curvature keeping $R_{y}$ constant, it is possible to optimize $R_{y}$, in order to increase the Laue lens performance. It is also possible to achieve a QM curvature equal to or larger than the constrained primary curvature.

To demonstrate that it is possible to control the QM curvature by setting $M_{1} \neq M_{2} \neq 0$, we report here the outcome of a recent experiment carried out using hard X-rays at the ID15A line of the European Synchrotron Radiation Facility (ESRF, Grenoble, France). An Si crystal of size $24 \times$ $62 \times 2 \mathrm{~mm}$ was deformed using a appropriately manufactured holder, capable of imparting two independent mechanical moments to the sample. The sample and holder were manufactured at the Sensor and Semiconductor Laboratory (SSL,

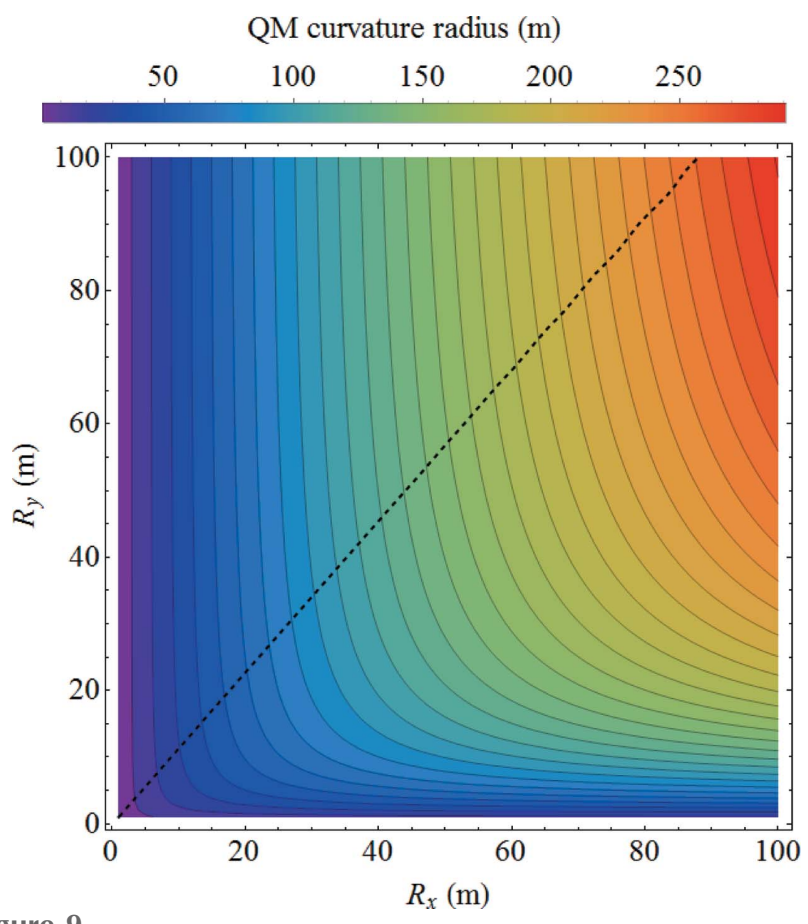

Figure 9

Radius of curvature due to the QM effect in a bent Si sample as a function of $R_{x}$ and $R_{y}$. The black dashed line represents the QM radius of curvature obtained with $M_{1}=M_{2}$. 
Table 3

Main features of the experiment.

\begin{tabular}{ll}
\hline Sample size $(\mathrm{mm})$ & $24 \times 62 \times 2$ \\
Main plane, orthogonal to the $z$ axis & $(211)$ \\
Planes orthogonal to the $x$ axis & $(111)$ \\
Planes orthogonal to the $y$ axis & $(110)$ \\
Beam energy $(\mathrm{keV})$ & 150 \\
Beam monochromaticity & $\Delta E / E=2 \times 10^{-3}$ \\
Beam divergency $\left(^{\prime \prime}\right)$ & $\sim 1$ \\
Beam size $(\mu \mathrm{m})$ & $50 \times 50$ \\
\hline
\end{tabular}

Ferrara, Italy). Fig. 10(a) shows a photograph of the sample, with the crystallographic orientations highlighted.

The holder was made of aluminium 7075. It kept the sample fixed and exploited flexure mechanisms to impart a highly controllable deformation to the crystal. In particular, two pairs of screws fixed the curvature of the holder, which transmitted the moments to the crystal. Fig. 10(b) shows a photograph of the sample.

A monochromatic and collimated beam was tuned at $150 \mathrm{keV}$. The monochromator was a fixed-exit double bent $\mathrm{Si}(111)$ Laue monochromator with an energy resolution of $\Delta E / E=2 \times 10^{-3}$. The beam was $50 \times 50 \mu \mathrm{m}$ wide. Sample characterization was carried out by performing rocking curves, i.e. by recording the diffracted beam intensity while the crystal was being rotated in the beam around the position where the Bragg condition was satisfied. The full width at half-maximum (FWHM) of the rocking curve was a direct measurement of the angular distribution of the diffracting planes. In this way, the radii of curvature along the $x, y$ and $z$ axes were measured. The experimental features are listed in Table 3.

The sample curvature was evaluated for three different dispositions. First disposition: the beam entered the sample through the $2 \times 62 \mathrm{~mm}$ face and diffracted onto the (211) planes. The traversed length of the sample $(24 \mathrm{~mm})$ divided by

(a)
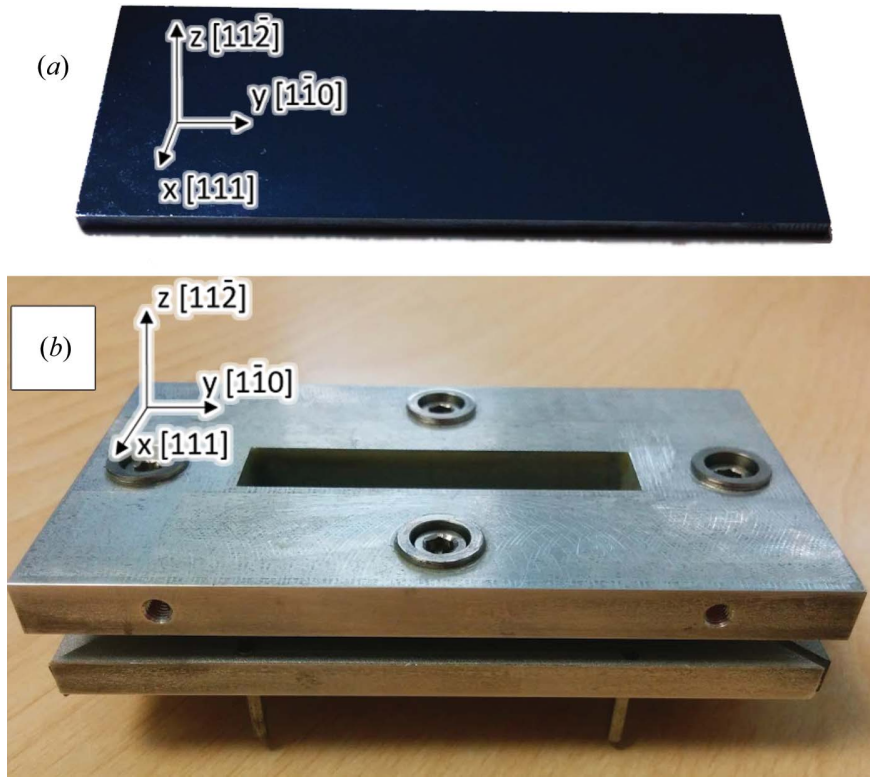

Figure 10

(a) Photograph of the QM Si sample. (b) Photograph of the holder, lateral view. Crystallographic orientations are highlighted. the FWHM of the obtained rocking curve was the radius of curvature along the $x$ axis, namely $R_{x}$. Second disposition: the beam entered the crystal through the $2 \times 24 \mathrm{~mm}$ face, traversing it along the longest side and diffracting onto the (211) planes. From this measurement, the radius of curvature $R_{y}$ along the $y$ axis was achieved. Third disposition: the sample was exposed to X-rays to measure the spread of the planes bent by the QM effect. The traversed face was the $24 \times$ $62 \mathrm{~mm}$, and the diffracting planes were the (111) planes.

Four different configurations were examined by exposing the crystal to X-rays, by setting four different curvatures for the sample. The configurations were obtained by turning the screws that controlled the sample deformation. At each configuration, the sample curvatures were measured in the three dispositions described above, to determine $R$ along the $x$ and $y$ axes and $R_{\mathrm{QM}}$ along the $z$ axis.

Using equations (5)-(10), it is possible to calculate the expected values for the QM curvature once the two values $R_{x}$ and $R_{y}$ are known. The theoretical values were compared with the measured ones and are plotted in Fig. 11. In the figure, the dashed lines show the theoretical QM curvature. The values are expressed as curvature $\left(C=1 / R \mathrm{~m}^{-1}\right)$ for better clarity of the data.

The four cases corresponding to experimental data are shown. The curvature along the $y$ axis is fixed, while that along the $x$ axis is an independent variable. The QM curvature is a function of the curvature along the $x$ axis. For each case, an experimental point was achieved, measuring the principal curvature along the $x$ and $y$ axes and the QM curvature. The experimental data are plotted as colored squares in Fig. 11.

A good agreement between the experimental data and the expected values of the QM curvature was achieved. This means that an adjustable value for the curvature of the planes bent by the QM effect can be achieved by controlling the primary curvature of a crystal. The black point in Fig. 11 corresponds to a radius of curvature of about $55 \mathrm{~m}$; this represents a very large curvature involving the QM effect in a 2 mm-thick crystal.

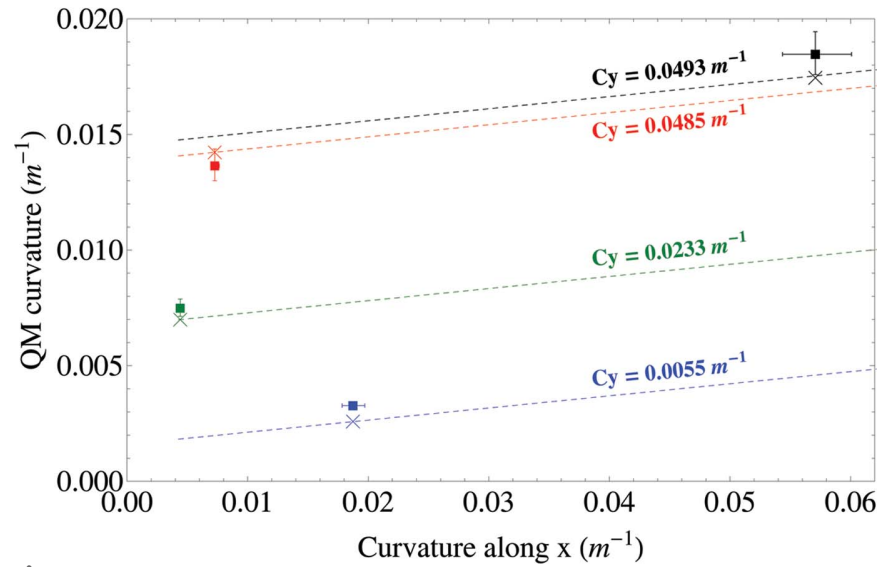

Figure 11

QM curvature in a deformed Si crystal. Squares represent experimental data and crosses represent expected values of the QM curvature. Dashed lines indicate different values of the curvature along the $y$ axis, while the values of curvature along the $x$ axis are shown by the abscissa axis. 


\section{Discussion and conclusions}

'Quasi-mosaicity' is an effect driven by anisotropy that was introduced 60 years ago. Half a century after its discovery, 'quasi-mosaicity' was revisited by the scientific community for two important applications in modern physics. First, the QM effect has been used for steering charged-particle beams with energy equal to or greater than $1 \mathrm{GeV}$. QM crystals have also proved to be an indispensable tool in the manipulation of negatively charged particle beams in the $\mathrm{GeV}$ energy range. Moreover, bent crystals based on the QM effect offer several opportunities for further research. For instance, steering of up to $10 \mathrm{GeV}$ electrons is currently underway at SLAC, while the electromagnetic radiation generated through interaction between $1 \mathrm{GeV}$ electrons and QM crystals is currently being studied at MAMI.

Second, 'quasi-mosaicity' may be a very promising effect for the focusing of hard X-rays and soft $\gamma$-rays. Two Laue lenses entirely based on QM crystals have been designed and proposed to the astrophysics community. The sensitivity of such Laue lenses was found to be very high when compared with other proposed lenses. Moreover, a Laue lens prototype relying on QM crystals is under development at the LARIX facility as part of the Laue project.

'Quasi-mosaicity' can be exploited for applications beyond those described in this paper. As an example, QM crystals are currently being studied for use as optical components for $\mathrm{X}$-ray monochromators. This scheme would allow the exploitation of curved diffracting planes, thus increasing the integrated diffraction efficiency of X-ray beams in synchrotron facilities or in diffractometers.

QM curvature can be exploited also in asymmetric lattice planes, i.e. in planes not parallel to any morphological surface. Actually, the theory of linear elasticity envisages that it is not possible to induce a QM curvature to several symmetric lattice planes. However, a QM crystal can show a curvature driven by anisotropy along asymmetric planes too. Indeed, some families of lattice planes that would be flat in a symmetric configuration can be bent under an asymmetric configuration. This condition is important because there are planes, such as the (220) family, that exhibit high electron density and thereby strong diffracting power but no QM effect under symmetric configurations. Indeed, the (220) planes can be bent through the QM effect if they were in an asymmetric configuration (Bellucci et al., 2015).

In this paper, we have reviewed the concept of 'quasimosaicity' in crystals from several points of view, encompassing its theoretical background and the technology behind the method. Finally, we have described a general case of two independent mechanical moments applied to a crystal plate to obtain an adjustable QM curvature. We performed an experiment at ESRF to demonstrate that the proposed treatment can predict the QM curvature as a function of the principal curvatures of the plate.

\section{Acknowledgements}

The authors are thankful to INFN for financial support through the LOGOS and CHANEL projects. T. Buslaps is gratefully acknowledged for technical assistance at line ID15A of ESRF.

\section{References}

Afonin, A. G., Baranov, V., Bellucci, S. et al. (2011). Instrum. Exp. Tech. 54, 1-7.

Afonin, A. G., Baranov, V. T., Biryukov, V. M. et al. (2001). Phys. Rev. Lett. 87, 094802.

Akbari, H. et al. (1993). Phys. Lett. B, 313, 491-497.

Authier, A. (2001). Dynamical Theory of X-ray Diffraction. Oxford University Press.

Authier, A. \& Malgrange, C. (1998). Acta Cryst. A54, 806-819.

Bagli, E., Bandiera, L., Bellucci, V., Berra, A., Camattari, R., De Salvador, D., Germogli, G., Guidi, V., Lanzoni, L., Lietti, D., Mazzolari, A., Prest, M., Tikhomirov, V. \& Vallazza, E. (2014). Eur. Phys. J. C, 74, 3114.

Baier, V. N., Katkov, V. M. \& Strakhovenko, V. M. (1998). Electromagnetic Processes at High Energies in Oriented Single Crystals. Singapore: World Scientific.

Bandiera, L., Mazzolari, A., Bagli, E., Berra, A., Lietti, D., De Salvador, D., Guidi, V., Prest, M., Tikhomirov, V. \& Vallazza, E. (2014). J. Phys. Conf. Ser. 517, 012043.

Barrière, N., Guidi, V., Bellucci, V., Camattari, R., Buslaps, T., Rousselle, J., Roudil, G., Arnaud, F.-X., Bastie, P. \& Natalucci, L. (2010). J. Appl. Cryst. 43, 1519-1521.

Bellazzini, R., Brez, A. \& Busso, L. (1991). Technical Reports CERNDRDC-91-26, CERN-PPE-91-26, DRDC-S-29 and DRCD-S-29. CERN, Geneva, Switzerland.

Bellucci, S., Bini, S. et al. (2003). Phys. Rev. Lett. 90, 034801.

Bellucci, V., Camattari, R. \& Guidi, V. (2013). Astron. Astrophys. 560, $1-8$.

Bellucci, V., Camattari, R., Guidi, V. \& Mazzolari, A. (2011). Thin Solid Films, 520, 1069-1073.

Bellucci, V., Camattari, R., Guidi, V., Neri, I. \& Barrière, N. (2011). Exp. Astron. 31, 45-58.

Bellucci, V., Paternò, G., Camattari, R., Guidi, V., Jentschel, M. \& Bastie, P. (2015). J. Appl. Cryst. 48, 297-300.

Bethe, W. H. (1934). Proc. R. Soc. London, 146, 83-112.

Biryukov, V. M., Chesnokov, Y. A., Guidi, V., Kotov, V. I., Malagú, C., Martinelli, G., Scandale, W., Stefancich, M. \& Vincenzi, D. (2002). Rev. Sci. Instrum. 73, 3170-3173.

Biryukov, V., Chesnokov, Y. A. \& Kotov, V. I. (1997). Crystal Channeling and Its Application at High-Energy Accelerators. Berlin: Springer.

Brejnholt, N. F., Soufli, R., Descalle, M., Fernández-Perea, M., Christensen, F. E., Jakobsen, A. C., Honkimäki, V. \& Pivovaroff, M. J. (2014). Opt. Express, 22, 15364-15369.

Brodsky, S., Fleuret, F., Hadjidakis, C. \& Lansberg, J. (2013). Phys. Rep. 522, 239-255.

Camattari, R., Battelli, A., Bellucci, V. \& Guidi, V. (2013). Exp. Astron. 37, 1-10.

Camattari, R., Bellucci, V., Guidi, V. \& Neri, I. (2011). Proc. SPIE, 8147, 81471G.

Camattari, R., Dolcini, E., Bellucci, V., Mazzolari, A. \& Guidi, V. (2014). J. Appl. Cryst. 47, 1762-1764.

Camattari, R., Guidi, V., Bellucci, V., Neri, I. \& Jentschel, M. (2013). Rev. Sci. Instrum. 84, 053110.

Camattari, R., Guidi, V., Lanzoni, L. \& Neri, I. (2013). Meccanica, 48, 1875-1882.

Camattari, R., Lanzoni, L., Bellucci, V. \& Guidi, V. (2015). J. Appl. Cryst. 48, 943-949.

Camattari, R., Paternò, G., Battelli, A., Bellucci, V., Bastie, B. \& Guidi, V. (2014). J. Appl. Cryst. 47, 799-802.

Camattari, R., Paternò, G., Bellucci, V. \& Guidi, V. (2014). Exp. Astron. 38, 417-431.

Carassiti, V., Dalpiaz, P., Guidi, V., Mazzolari, A. \& Melchiorri, M. (2010). Rev. Sci. Instrum. 81, 066106. 
Carrigan, R. et al. (2002). Phys. Rev. ST Accel. Beams, 5, 043501.

Denisov, A., Fedin, O., Gordeeva, M., Gur'ev, M., Platonov, Y., Schetkovsky, A., Skorobogatov, V., Smirnov, A., Baranov, V., Chesnokov, Y., Dudenko, V., Galyaev, N., Kotov, V., Tsarik, S. \& Zapolsky, V. (1992). Phys. Res. B, 69, 382-384.

Elishev, A. et al. (1979). Phys. Lett. B, 88, 387-391.

Feldman, L., Mayer, J. \& Picraux, S. (1982). Materials Analysis by Ion Channeling: Submicron Crystallography. St Louis: Academic Press.

Fernández-Perea, M., Descalle, M.-A., Soufli, R., Ziock, K. P., Alameda, J., Baker, S. L., McCarville, T. J., Honkimäki, V., Ziegler, E., Jakobsen, A. C., Christensen, F. E. \& Pivovaroff, M. J. (2013). Phys. Rev. Lett. 111, 027404.

Ferrari, C., Buffagni, E., Bonnini, E. \& Korytar, D. (2013). J. Appl. Cryst. 46, 1576-1581.

Fliller, R. III, Drees, A., Gassner, D., Hammons, L., McIntyre, G., Peggs, S., Trbojevic, D., Biryukov, V., Chesnokov, Y. \& Terekhov, V. (2005). Phys. Res. B, 234, 47-56.

Frontera, F. et al. (2012). Proc. SPIE, 8443, 84430B.

Guidi, V., Barrière, N., Bellucci, V., Camattari, R. \& Neri, I. (2011). Proc. SPIE, 8147, 81471E.

Guidi, V., Bellucci, V., Camattari, R. \& Neri, I. (2011). J. Appl. Cryst. 44, 1255-1258.

Guidi, V., Lanzoni, L. \& Mazzolari, A. (2010). J. Appl. Phys. 107, 113534.

Guidi, V., Lanzoni, L. \& Mazzolari, A. (2011). Thin Solid Films, 520, 1074-1079.

Guidi, V., Mazzolari, A., De Salvador, D. \& Carnera, A. (2009). J. Phys. D Appl. Phys. 42, 182005.

Ivanov, Y., Bondar, N., Gavrikov, Y., Denisov, A., Zhelamkov, A., Ivochkin, V., Kosyanenko, S., Lapina, L., Petrunin, A., Skorobogatov, V., Suvorov, V., Shchetkovsky, A., Taratin, A. \& Scandale, W. (2006). JETP Lett. 84, 372-376.

Ivanov, Y., Petrunin, A. \& Skorobogatov, V. (2005). JETP Lett. 81, 99-101.

Korol, A. V., Solov'yov, A. V. \& Greiner, W. (2004). Int. J. Modern Phys. E, 13, 867-916.

Kumakhov, M. (1976). Phys. Lett. A, 57, 17-18.

Lekhnitskii, S. (1981). Theory of Elasticity of an Anisotropic Body. Moscow: Mir Publishers.

Lekhnitskii, S., Tsai, S. \& Cheron, T. (1956). Anisotropic Plates. New York: Gordon and Breach Science Publishers.

Liccardo, V., Virgilli, E., Frontera, F., Valsan, V., Buffagni, E., Ferrari, C., Bonnini, E., Zappettini, A., Guidi, V., Bellucci, V. \& Camattari, R. (2014). Exp. Astron. 38, 401-416.

Lind, D. A., West, W. J. \& DuMond, J. W. M. (1950). Phys. Rev. 77, 475-490.

Lindhard, J. (1965). Danske Vid. Selsk. Mater. Fys. Medd. 34, 1-64.

Loffredo, G., Frontera, F., Pellicciotta, D., Pisa, A., Carassiti, V., Chiozzi, S., Evangelisti, F., Landi, L., Melchiorri, M. \& Squerzanti, S. (2005). Exp. Astron. 20, 413-420.

Lohmann, D. et al. (1994). Nucl. Instrum. Methods Phys. Res. Sect. A, 343, 494-507.

Lund, N. (1992). Exp. Astron. 2, 259-273.

Maslov, M., Mokhov, N. \& Yazynin, I. (1991). Techical Report SSCL 484. Superconducting Super Collider, Waxahachie, Texas, USA.

Mazzolari, A., Bagli, E., Bandiera, L., Guidi, V., Backe, H., Lauth, W., Tikhomirov, V., Berra, A., Lietti, D., Prest, M., Vallazza, E. \& De Salvador, D. (2014). Phys. Rev. Lett. 112, 135503.
Mazzolari, A., Camattari, R., Bellucci, V., Paternò, G., Scian, C., Mattei, G. \& Guidi, V. (2015). Nucl. Instrum. Methods Phys. Res. Sect. B, 355, 297-300.

Mikula, P., Krüger, E., Scherm, R. \& Wagner, V. (1990). J. Appl. Cryst. 23, 105-110.

Paternò, G., Bellucci, V., Camattari, R. \& Guidi, V. (2015). J. Appl. Cryst. 48, 125-137.

Piercy, G. R., Brown, F., Davies, J. A. \& McCargo, M. (1963). Phys. Rev. Lett. 10, 399-400.

Popovici, M., Stoica, A. D., Yelon, W. B. \& Berliner, R. (1999). Proc. SPIE, 3767, 320-327.

Rekveldt, M. (1983). Nucl. Instrum. Methods Phys. Res. 215, 521527.

Rekveldt, M. T. \& Westerhuuijs, P. (1987). Nucl. Instrum. Methods Phys. Res. Sect. B, 28, 583-591.

Roa, D., Smither, R., Zhang, X., Nie, K., Shieh, Y., Ramsinghani, N., Milne, N., Kuo, J., Redpath, J., Al-Ghazi, M. \& Caligiuri, P. (2005). Exp. Astron. 20, 229-239.

Scandale, W., Arduini, G., Assmann, R., Bracco, C. et al. (2010). Phys. Lett. B, 692, 78-82.

Scandale, W., Arduini, G., Assmann, R., Losito, R., Cavoto, G., Ivanov, Y.-M., Hall, G., Markiewicz, T., Taratin, A.-M. \& Chesnokov, Y.-A. (2011). Technical Reports CERN-LHCC-2011007 and LHCC-I-019. CERN, Geneva, Switzerland.

Scandale, W., Carnera, A. et al. (2008a). Phys. Lett. B, 658, 109-111.

Scandale, W., Carnera, A. et al. (2008b). Phys. Rev. ST Accel. Beams, 11, 063501.

Scandale, W., Losito, R. et al. (2013). Phys. Lett. B, 719, 70-73.

Scandale, W., Vomiero, A., Bagli, E. et al. (2009). Phys. Lett. B, 681, 233-236.

Scandale, W., Vomiero, A., Baricordi, S. et al. (2009). Phys. Rev. A, 79, 012903.

Schulze, C., Lienert, U., Hanfland, M., Lorenzen, M. \& Zontone, F. (1998). J. Synchrotron Rad. 5, 77-81.

Shiltsev, V. et al. (2010). Proceedings of the 1st International Particle Accelerator Conference: IPAC'10, pp. 4398-4400. ACFA.

Smither, R. K., Saleem, K. A., Roa, D. E., Beno, M. A., Ballmoos, P. V. \& Skinner, G. K. (2005). Exp. Astron. 20, 201-210.

Sumbaev, O. (1957). Soviet Phys. JETP, 27, 1042-1044.

Sumbaev, O. (1968). Soviet Phys. JETP, 5, 724-728.

Suortti, P., Lienert, U. \& Schulze, C. (1997). AIP Conf. Proc. 389, 175192.

Tabrizi, M., Korol, A. V., Solov'yov, A. V. \& Greiner, W. (2007). Phys. Rev. Lett. 98, 164801.

Taratin, A. M. \& Vorobiev, S. A. (1987). Phys. Lett. A, 119, 425-428.

Tsyganov, E. N. (1976a). Preprint TM-684. Fermilab, Batavia, IL, USA.

Tsyganov, E. N. (1976b). Preprint TM-682. Fermilab, Batavia, IL, USA.

Virgilli, E., Frontera, F., Valsan, V., Liccardo, V., Carassiti, V. et al. (2013). Proc. SPIE, 8861, 886107.

Virgilli, E., Frontera, F., Valsan, V., Liccardo, V., Caroli, E., Stephen, J. B., Cassese, F., Recanatesi, L., Pecora, M., Mottini, S., Attinà, P. \& Negri, B. (2011). Proc. SPIE, 8147, 81471C.

Von Ballmoos, P. (2013). Nucl. Instrum. Methods Phys. Res. Sect. B, 309, 244-248.

Zachariasen, W. H. (1945). Theory of X-ray Diffraction in Crystals. New York: J. Wiley and Sons. 